\title{
Characteristics of minor head trauma in toddlers
}

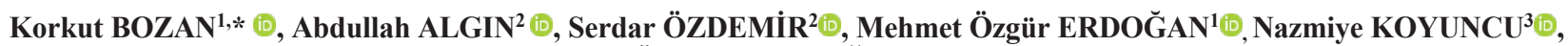 \\ Özgür KARCIOĞLU ${ }^{4}$ (D) \\ ${ }^{1}$ Department of Emergency Medicine, Göztepe Medical Park Hospital, İstanbul, Turkey \\ ${ }^{2}$ Department of Emergency Medicine, University of Health Sciences Turkey Umraniye Training and Research Hospital, Istanbul \\ ${ }^{3}$ Department of Emergency Medicine, University of Healty Science, Haydarpaşa Numune Training and Research Hospital, İstanbul, Turkey \\ ${ }^{4}$ Department of Emergency Medicine, University of Healty Science, İstanbul Training and Research Hospital, İstanbul, Turkey
}

\begin{abstract}
Received: $22.03 .2021 \quad \bullet \quad$ Accepted/Published Online: $27.03 .2021 \quad \bullet \quad$ Final Version: 30.08 .2021
Abstract

The objective of present study is to evaluate mechanisms and causes of head trauma, factors influencing management of pediatric minor head trauma, to highlight decision making processes in diagnostic imaging as well as searching for preventive measures for head trauma. Children younger than two years of age who were admitted to emergency department in one-year study period due to minor head trauma were included to the study. To be inside or outside of house did not significantly change the incidence of falls for children younger than two years of age $(\mathrm{p}=0.096)$. Incidence of falls was significantly increased at living rooms $(\mathrm{p}=0.01)$ and bathrooms $(\mathrm{p}=0.036)$. Incidence of scalp hematomas was significantly higher in symptomatic patients $(\mathrm{p}=0.006)$. Asymptomatic admission after a minor injury was not a significant factor on decision of diagnostic imaging. A patient's asymptomatic presentation should not be used as a criterion to rule out cerebral injuries. A lack of obvious signs and symptoms during evaluation does not exclude TBI. Existing serious symptoms should lead to a quick evaluation of patient to rule out a possible surgical emergency. Scalp hematomas are significantly associated with cerebral injury and are a predictor of brain injury. Clinicians should have a lower threshold for imaging in children $<2$ years of age.
\end{abstract}

Keywords: children, epidemiology, head trauma, imaging

\section{Introduction}

Trauma is the most common cause of death in children and the fifth most common cause of death in adults. Up to half of mortalities related to trauma are secondary to head injuries (1, 2). Children are more vulnerable to head trauma due to their larger heads relatively to their body size, incomplete brain myelination, and incomplete closure of the cranial sutures (1, 2).

Management of minor pediatric head trauma and the choice of diagnostic imaging modality are commonly based on the risk of traumatic brain injury (TBI) and age subcategory. Head trauma is a clinical challenge for healthcare providers in children $<2$ years of age (3). Clinical assessment is difficult in this age group, as these patients cannot describe the mechanism of the injury. In some cases, accidental or non-accidental causes of injury cannot be clearly identified (4, 5). In addition, anatomical differences in children $<2$ years of age pose a major problem and may lead to an asymptomatic presentation with subtle findings and/or late onset of symptoms (3).

In the present study, clinical and demographic characteristics of toddlers $(<2$ years of age) who were admitted to the emergency department (ED) for minor head trauma were evaluated. This study collected all injury data to highlight factors that may affect the outcomes of minor head trauma in toddlers. We investigated the mechanisms and causes of head trauma and the factors influencing the management of symptomatic and asymptomatic minor pediatric head trauma to highlight the choice of diagnostic imaging modality and search for preventive measures for head trauma.

\section{Materials and methods}

This prospective observational study was initiated following approval from the Institutional Review Board (HNEAH KAEK2013/275). Ethics committee approval was received for this study from the ethics committee of University of Health Sciences, Haydarpasa Training and Research Hospital (HNEAH KAEK2013/275). All consecutive children aged $<2$ years who were admitted to the ED during the one-year study period for minor head trauma were included in the study. Patients with no clear history of trauma, those $>2$ years of age, those who had returned for reassessment of the same head trauma, victims of child abuse, children with chronic 
diseases, and children whose parents refused to provide informed written consent were excluded from the study. All patients were followed for 12 hours in the ED observation room. Data about history of the trauma and symptoms were obtained from the parents and/or relatives of the children. Age and physical examination and radiological findings were recorded.

The choice of diagnostic imaging modality was based on the Pediatric Emergency Care Applied Research Network (PECARN) (6). Computed tomography (CT) scans of the head were examined by radiologists and were considered positive for TBI if pathological findings such as skull fracture or subdural, epidural, or subarachnoid bleeding were apparent. All head CT scans were re-evaluated by a senior radiologist, and no disagreement occurred.

\subsection{Statistical analysis}

The patients were divided into symptomatic and asymptomatic groups. Data were analyzed using SPSS ver. 17.0 software (SPSS, Inc., Chicago, Il, USA). Categorical variables were analyzed with Fisher's exact test, and continuous variables were analyzed with the independent sample $t$-test. The level of significance was set at 0.05 .

\section{Results}

A total of 551 children aged $<2$ years were admitted to the ED during the study period. Among them, 48 were excluded from the study due to a lack of written informed consent. Thus, 503 patients were included in the study group. The mean age of all children was $12.4 \pm 7.0$ months. The mean age of the 234 (46.5\%) girls was $12.1 \pm 6.9$ months, and the mean age of the $269(53.5 \%)$ boys was $12.6 \pm 7.1$ months $(p=0.423)$. The age and sex distribution are shown in Table 1.

Table 1. Gender and age distribution

\begin{tabular}{|c|c|c|c|} 
& Female & Male & Total \\
\hline $0-1$ years & $138(27.4 \%)$ & $145(28.8 \%)$ & $283(56.3 \%)$ \\
\hline $1-2$ years & $96(19 \%)$ & $124(24.6 \%)$ & $220(43.7 \%)$ \\
\hline
\end{tabular}

The most common mechanism of injury was falls, seen in $184(36.5 \%)$ patients; falls from height were recorded in 26 (5.1\%) patients, $34(6.7 \%)$ children fell while a parent was holding them, objects fell on $20(3.9 \%)$ children, $36(7.1 \%)$ patients collided with an object, $2(0.4 \%)$ were in vehicle accidents, $2(0.4 \%)$ were in pedestrian accidents, $14(2.7 \%)$ fell from a baby walker, $14(2.7 \%)$ were injured by next of kin, $70(13.9 \%)$ fell from a cradle, $41(8.1 \%)$ fell from an adult bed, $40(7.9 \%)$ fell from a couch/furniture, and 18 (3.5\%) patients fell from a stroller. The distribution of the mechanisms of injury is shown at Table 2.

Ground-level falls inside the home were the most common type of injury (126 [68.4\%] children), and 58 (31.5\%) children fell outside the home. The location of the injury (inside vs. outside the home) did not significantly affect the incidence of falls $(p=0.096)$.
Table 2. Distribution of the mechanisms of injuries

\begin{tabular}{|l|c|}
\hline \multicolumn{1}{|c|}{ Mechanism of injury } & N (\%) \\
\hline Ground-level falls & $184(36.5 \%)$ \\
\hline Falls from heights & $26(5.1 \%)$ \\
\hline Parent fall while holding children & $34(6.7 \%)$ \\
\hline Object falls on children & $20(3.9 \%)$ \\
\hline Vehicle accident & $2(0.3 \%)$ \\
\hline Pedestrian accident & $2(0.3 \%)$ \\
\hline Fall from baby walker & $14(2.7 \%)$ \\
\hline Sibling caused injuries & $14(2.7 \%)$ \\
\hline Falls from cradles/baby beds & $70(13.9 \%)$ \\
\hline Falls from adult beds & $41(8.1 \%)$ \\
\hline Falls from couches & $40(7.9 \%)$ \\
\hline Collision with an object & $36(7.1 \%)$ \\
\hline Falls from strollers & $18(3.5 \%)$ \\
\hline Unknown & $2(0.3 \%)$ \\
\hline Total & $503(100 \%)$ \\
\hline
\end{tabular}

The incidence of falls was significantly higher in the living room $(p=0.01)$ and bathroom $(p=0.036)$ compared to other parts of the home, such as the kitchen, hallway, or baby room. The most common injury locations due to falls and other injuries inside the home are shown in Table 3.

A total of $408(81.1 \%)$ asymptomatic patients were included in the study group. No diagnostic images were taken in $16(3.9 \%)$ of these patients. Patients who presented with symptoms underwent diagnostic imaging. Admission after a minor asymptomatic injury was not a significant factor when choosing the diagnostic imaging modality.

A repeat CT scan was obtained in 14 of 408 asymptomatic patients and in 14 of 95 symptomatic patients on admission. These repeat CT scans were performed in patients with new or persistent symptoms during the observation period. No repeat CT scan was taken after an asymptomatic observation $\operatorname{period}(\mathrm{p}=0.0001)$.

Twelve $(12.6 \%)$ of 95 symptomatic patients and 18 (4.4\%) asymptomatic patients had scalp hematomas. The incidence of scalp hematomas was significantly higher in symptomatic than in asymptomatic patients $(p=0.006)$.

Thirteen $(13.6 \%)$ of the 95 symptomatic patients and 10 (2.4\%) of 408 asymptomatic patients had fractures detected on a CT scan. Cranial fractures were significantly associated with a symptomatic admission $(\mathrm{p}=0.000)$, and $2.4 \%$ of asymptomatic patients also had cranial fractures.

Eight $(8.4 \%)$ of the 95 symptomatic patients and 4 $(0.98 \%)$ of the 408 asymptomatic patients had epidural hematomas on CT imaging. Epidural hematomas were significantly associated with the symptomatic admissions ( $\mathrm{p}=$ $0.0003)$. 
Table 3. Common injury locations of falls and other injuries inside the houses

\begin{tabular}{|c|c|c|c|}
\hline $\begin{array}{l}\text { Injury } \\
\text { Location }\end{array}$ & Falls n (\%) & Other n (\%) & $\mathbf{p}$ \\
\hline Living room & $62(33.6 \%)$ & $64(20.1)$ & 0.01 \\
\hline Bathroom & $10(5.4 \%)$ & $6(1.8 \%)$ & 0.036 \\
\hline Hallway & $8(4.3 \%)$ & $18(8.6 \%)$ & 0.677 \\
\hline Baby room & $30(16.3 \%)$ & $84(26.3 \%)$ & 0.011 \\
\hline Kitchen & $4(2.1 \%)$ & $18(5.6 \%)$ & 0.073 \\
\hline Balcony & $6(3.2 \%)$ & $8(2.5 \%)$ & 0.53 \\
\hline $\begin{array}{l}\text { Adult } \\
\text { bedroom }\end{array}$ & $6(3.2 \%)$ & $43(13.4 \%)$ & 0.008 \\
\hline $\begin{array}{l}\text { Total in } \\
\text { house }\end{array}$ & $126(68.4 \%)$ & $241(75.54 \%)$ & \\
\hline Total & $184(100 \%)$ & $319(100 \%)$ & \\
\hline
\end{tabular}

Six $(6.3 \%)$ of 95 symptomatic patients and four $(0.98 \%)$ of 408 asymptomatic patients had cerebral contusions on CT imaging. Cerebral contusions were significantly associated with symptomatic admissions $(\mathrm{p}=0.004)$. Four $(4.2 \%)$ patients with intracerebral hemorrhages were in the symptomatic group, and none was in the asymptomatic group. Two patients in the study group had pneumocephalus, and all of these patients were in the symptomatic group. No cerebral edema was reported in the study group.

Eighty-nine (17.6\%) of 503 patients consulted with neurosurgeons. The frequency of consultations was significantly higher in symptomatic than in asymptomatic patients $(\mathrm{p}=0.0001)$. Twenty-three $(4.5 \%)$ patients were hospitalized, and symptomatic patients were more commonly hospitalized $(\mathrm{p}=0.000)$ than asymptomatic patients. Three patients in the symptomatic group underwent surgery. Six $(1.2 \%)$ patients required a reassessment in the first 24 hours after discharge from the hospital.

\section{Discussion}

Falls were the most common cause of minor head trauma in this study group. Falls from a baby bed, an adult bed, or a couch, as well as falls while a parent was holding the child were common injury mechanisms. This high incidence of falls was related to toddlers' poor balance $(1,4)$. In the present study, the incidence of falls did not differ between those inside and outside the home in children $<2$ years of age. Toddlers generally live inside the home, and parents pay more attention when they are outside, which may have decreased the incidence of outdoor injuries $(7,8)$. Falls in the home were most common in the living room and bathroom. Protective measures against head injuries should be used in the living room and bathroom. These parts of the home have many possible risk factors that may lead to head injuries, and protective measures may decrease the incidence of head trauma $(8,9)$. Most head trauma cases are low-risk traumas. The clinical challenges when evaluating minor head trauma in children are to identify TBI and limit CT imaging and radiation exposure. A head $\mathrm{CT}$ scan is the gold standard for diagnosing patients with a head injury (10). Neuroimaging with CT is extremely sensitive for brain injury (10). However, clinical predictors of brain injury are commonly nonspecific in children aged $<2$ years $(5,6)$.

In the present study, symptomatic patients had a higher incidence of skull fractures, but these fractures were also seen in $2.45 \%$ of asymptomatic patients. Using initial symptoms as the sole predictor of TBI or as the basis of a choice of imaging modality has a high incidence of failure when trying to detect a fracture. In this study, the decision to obtain a head CT was not related to symptoms, physical examination findings, or clinical decision rules. Clinicians have a lower threshold for imaging in children $<2$ years of age (11).

Although PECARN is sensitive for this age group, treatment decisions depend on physical examination findings and the history taken from a parent (6). The CHALICE rules accept a wide range of patients, and the original article reported 98\% sensitivity for minor head trauma (5). Decision rules fail to provide a management algorithm for head trauma. These rules simply assist the clinician with their choice of whether to obtain a head CT scan. For these reasons, clinicians prefer imaging to rule out TBI in this age group. The decision to obtain a second head CT was based on observation. In this study, clinicians obtained a second head $\mathrm{CT}$ in patients with persistent or new symptoms $(\mathrm{p}=0.000)$.

Scalp hematomas are a common finding in pediatric cases of head trauma and are a sign for an increased risk of intracranial injury. Parietal, temporal, and occipital scalp hematomas should alert the physician to an increased risk of intracranial injury $(7,11,12)$. Recent studies reveal that intracranial injuries are associated with scalp hematomas in $93 \%$ of cases. In this study, symptomatic patients had a significantly higher incidence of scalp hematomas than did asymptomatic patients.

The incidence of epidural hematoma was significantly higher in symptomatic than that in asymptomatic patients. However, an epidural hematoma cannot be ignored in an asymptomatic patient, and asymptomatic presentation does not rule out an epidural hematoma. No subdural hematomas were detected in the study group, so the present study could not reveal the features of subdural hematomas in this age group. Subdural hematomas should be further evaluated in a future study. Symptomatic patients had a significantly higher incidence of cerebral contusions than did the asymptomatic group in our study. All intracerebral hemorrhages were in the symptomatic group. Although all these diagnoses were more common in the symptomatic group, they were noted with a lower incidence in the asymptomatic group. Symptoms alone should not be used to predict a cerebral injury.

A patient's asymptomatic presentation should not be used 
as a criterion to rule out a cerebral injury. Management of head injury in this age group should not be based solely on presenting symptoms. A lack of obvious signs and symptoms during the evaluation does not exclude TBI. Existing serious symptoms should lead to a quick evaluation of the patient to rule out a possible surgical emergency. Scalp hematomas are significantly associated with cerebral injury and are a predictor of brain injury. Physicians should be aware that decision rules do not provide an algorithm for managing head trauma (12). These rules are a guide for the clinician's decision about whether to obtain a head CT scan. Clinicians should have a lower threshold for imaging in children $<2$ years of age $(3,12-14)$. Head injuries in children aged $<2$ years are commonly due to preventable injuries (3). Family education may be effective and can contribute to lowering the incidence of these injuries (15). For example, family members should be aware that a baby can turn over after four months. Significant results can be realized by modifying the physical environment. Falls in the home were common in living rooms and bathrooms. Shock-absorbent floors or protective mats may reduce the risk of injury in homes. Similarly, protective measures should be taken against head injuries in living rooms and bathrooms (15). As the latency period for developing a malignancy may be decades, mortality due to malignancies caused by head $\mathrm{CT}$ radiation are often ignored. The incidence of head and neck malignancies caused by head CT radiation should be clarified (14). Further studies should focus on improving the sensitivity of clinical decision rules and designing a universal algorithm for managing minor head trauma in children aged $<2$ years.

Our study has several limitations. Because our data were collected in a tertiary hospital, they may not represent the entire population. We did not evaluate protective measures in the home. A standard decision rule could not be chosen due to the observational design of the study. Some injuries, such as subdural hematomas or brain edema, were not noted in the study group, and 48 patients were excluded from the study due to a lack of written informed consent. Excluding many patients from the study may have influenced the results.

A patient's asymptomatic presentation should not be used as a criterion to rule out a cerebral injury. Management of head injury in this age group should not be based solely on presenting symptoms. A lack of obvious signs and symptoms during the evaluation does not exclude TBI. Existing serious symptoms should lead to a quick evaluation of the patient to rule out a possible surgical emergency. Scalp hematomas are significantly associated with cerebral injury and are a predictor of brain injury. Clinicians should have a lower threshold for imaging in children $<2$ years of age.

\section{Conflict of interest}

The authors have no conflict of interests to declare.

\section{Acknowledgments}

Written and verbal informed consent was obtained from patients who participated in this study. We would like to thank Textcheck for English language editing. A native speaker edited the language of the manuscript. For a certificate see http://www.textcheck.com/certificate/IXjxhC. The author declared that this study has received no financial support.

\section{References}

1. Hawley C, Wilson J, Hickson C, Mills S, Ekeocha S, Sakr M. Epidemiology of paediatric minor head injury: Comparison of injury characteristics with Indices of Multiple Deprivation. Injury. 2013; 44(12):1855-61. doi: 10.1016/j.injury.2013.07.021.

2. Hardelid P, Davey J, Dattani N, Gilbert R; Working Group of the Research and Policy Directorate of the Royal College of Paediatrics and Child Health. Child deaths due to injury in the four UK countries: a time trends study from 1980 to 2010. PLoS One. 2013 Jul 10;8(7):e68323. doi: 10.1371/journal.pone.0068323.

3. Schutzman SA, Barnes P, Duhaime AC, Greenes D, Homer C, Jaffe D, et al. Evaluation and management of children younger than two years old with apparently minor head trauma: proposed guidelines. Pediatrics. 2001;107(5):983-93. doi: 10.1542/peds.107.5.983.

4. Gordy C, Kuns B. Pediatric abusive head trauma. Nurs Clin North Am. 2013; 48(2):193-201. doi: 10.1016/j.cnur.2013.01.013.

5. Crowe L, Anderson V, Babl FE. Application of the CHALICE clinical prediction rule for intracranial injury in children outside the UK: impact on head CT rate. Arch Dis Child. 2010; 95(12):1017-22. doi: 10.1136/adc.2009.174854.

6. Kuppermann N, Holmes JF, Dayan PS, Hoyle JD Jr, Atabaki SM, Holubkov R, et al. ; Pediatric Emergency Care Applied Research Network (PECARN). Identification of children at very low risk of clinically-important brain injuries after head trauma: a prospective cohort study. Lancet. 2009 Oct 3;374(9696):1160-70. doi: 10.1016/S0140-6736(09)61558-0.

7. Maxwell WL. Traumatic brain injury in the neonate, child and adolescent human: an overview of pathology. Int J Dev Neurosci. 2012; 30(3):167-83. doi: 10.1016/j.ijdevneu.2011.12.008.

8. Siddiqui EU, Ejaz K, Siddiqui U. Unintentional, paediatric domestic injury in a semi rural area of Karachi. J Pak Med Assoc. 2012; 62(7):638-43.

9. Deans KJ, Thackeray J, Askegard-Giesmann JR, Earley E, Groner JI, Minneci PC. Mortality increases with recurrent episodes of nonaccidental trauma in children. J Trauma Acute Care Surg. 2013; 75(1):161-5. doi: 10.1097/ta.0b013e3182984831.

10. Mannix R, Meehan WP, Monuteaux MC, Bachur RG. Computed tomography for minor head injury: variation and trends in major United States pediatric emergency departments. J Pediatr. 2012; 160(1):136-9.e1. doi: 10.1016/j.jpeds.2011.06.024.

11. Anthikkat AP, Page A, Barker R. Risk factors associated with injury and mortality from paediatric low speed vehicle incidents: a systematic review. Int J Pediatr. 2013;2013:841360. doi: $10.1155 / 2013 / 841360$.

12. Pickering A, Harnan S, Fitzgerald P, Pandor A, Goodacre S. Clinical decision rules for children with minor head injury: a systematic review. Arch Dis Child. 2011; 96(5):414-21. doi: 10.1136/adc.2010.202820.

13. Hamilton M, Mrazik M, Johnson DW. Incidence of delayed intracranial hemorrhage in children after uncomplicated minor head injuries. Pediatrics. 2010; 126(1):e33-9. doi: 10.1542/peds.2009-0692. 
Özdemir et al. / J Exp Clin Med

14. Holmes JF, Borgialli DA, Nadel FM, Quayle KS, Schambam N, Cooper A, et al. ; TBI Study Group for the Pediatric Emergency Care Applied Research Network. Do children with blunt head trauma and normal cranial computed tomography scan results require hospitalization for neurologic observation? Ann Emerg
Med.

2011

$58(4): 315-22$.

doi: 10.1016/j.annemergmed.2011.03.060.

15. Höllwarth ME. Prevention of unintentional injuries: a global role for pediatricians. Pediatrics. 2013;132(1):4-7. doi: 10.1542/peds.2013-0571. 